\title{
Impulsive Control and Synchronization of Complex Lorenz Systems
}

\author{
Shaban Aly, ${ }^{1,2}$ Ali Al-Qahtani, ${ }^{1}$ Houari B. Khenous, ${ }^{1}$ and Gamal M. Mahmoud ${ }^{3,4}$ \\ ${ }^{1}$ Department of Mathematics, Faculty of Science, King Khalid University, P.O. Box 9004, Abha, Saudi Arabia \\ ${ }^{2}$ Department of Mathematics, Faculty of Science, Al-Azhar University, Assiut 71511, Egypt \\ ${ }^{3}$ Department of Mathematics, Faculty of Science, Assiut University, Assiut 71516, Egypt \\ ${ }^{4}$ Department of Mathematics, Faculty of Science, Taibah University, Al-Madinah Al-Munawarah, Saudi Arabia
}

Correspondence should be addressed to Shaban Aly; shhaly70@yahoo.com and Ali Al-Qahtani; alitalhan@hotmail.com

Received 6 February 2014; Accepted 16 March 2014; Published 28 April 2014

Academic Editor: Jun $\mathrm{Hu}$

Copyright (C) 2014 Shaban Aly et al. This is an open access article distributed under the Creative Commons Attribution License, which permits unrestricted use, distribution, and reproduction in any medium, provided the original work is properly cited.

\begin{abstract}
In this paper, we continue our investigations on control and synchronization of the complex Lorenz systems by investigating impulsive control and synchronization. Nonlinear systems involving impulse effects, appear as a natural description of observed evolution phenomena of several real world problems; For example, many biological phenomena involving thresholds, bursting rhythm models in medicine and biology, optimal control models in economics, population dynamics and so forth do exhibit impulsive effects. Some new and more comprehensive criteria for global exponential stability and asymptotical stability of impulsively controlled complex Lorenz systems are established with varying impulsive intervals. The effectiveness of the proposed technique is verified through numerical simulations.
\end{abstract}

\section{Introduction}

In the last 30 years, chaotic systems involving both real and complex variables governed by complex ordinary differential equations have been widely studied and investigated which contain one or more complex variables that make multiplication of variables that appear in many important applications in engineering; for example, in communications, where doubling the number of variables may be used to increase the content and security of the transmitted information, used to describe and simulate the physics of detuned laser and thermal convection of liquid flows, the electric field amplitude and the atomic polarization amplitude are both complex (see [1-5]).

In applied sciences and engineering, there are a lot of problems which are described by these complex systems, for example, in many important fields of physics, engineering, and computer science, such as laser physics, control, flow dynamics and liquid mixing, electronic circuits, secure communications, and information sciences (see [6-13]).
In 1963, Lorenz introduced the real Lorenz system (i.e., system involves real variables):

$$
\begin{gathered}
\dot{x}=a(y-x), \quad \dot{y}=c x-y-x z, \\
\dot{z}=-b z+x y,
\end{gathered}
$$

where $a>0, c>0$, and $b>0$. This system describes the thermal convection in fluids, problems of laser physics, and disk dynamos (see [7, 14-17]).

In recent years, there has been considerable interest in the control of chaos in nonlinear dynamical systems. For the past years, many different techniques have been proposed to control chaos, including Ott, Grebogi, and Yorke (OGY) method, Pecore and Carroll (PC) technique, and back stepping approach (see [18-22]).

As the key technology of secure communication, chaotic synchronization has been widely developed since Pecora and Carroll (see [18]) proposed the principle of chaos synchronization and realized it in the circuit in 1990. The basic behavior and chaotic synchronization of (1) have been 
studied by several researchers (see [16, 23-25]). The concept of synchronization consists in making two chaotic systems (identical or different) oscillate in a synchronized manner. A wide variety of methods have been proposed and applied for the synchronization of chaotic systems which include, for example, active control, global synchronization, adaptive control, linear and nonlinear feedback, and back stepping design (see $[16,25,26]$ and references therein).

Many evolution processes are characterized by the fact that at certain moments of time they experience a change of state abruptly. These processes are subject to short-term perturbations whose duration is negligible in comparison with the duration of the process. Consequently, it is natural to assume that these perturbations act instantaneously, that is, in the form of impulses. The impulsive differential equations, that is, differential equations involving impulse effects, appear as a natural description of observed evolution phenomena of several real world problems; for example, many biological phenomena involving thresholds, bursting rhythm models in medicine and biology, optimal control models in economics, and population dynamics do exhibit impulsive effects (see [27-31]). Recently, impulsive control has been widely used to stabilize and synchronize chaotic systems (see [32-34]). Its necessity and importance lie in the fact that, in some cases, the system cannot be controlled by continuous control. For example, a government cannot change savings rates of its central bank every day. Additionally, impulsive control may give a more efficient method to deal with systems that cannot endure continuous disturbance. Furthermore, impulsive method can also greatly reduce the control cost. A chaotic system is a nonlinear deterministic problem that displays complex and unpredictable behavior. Chaotic synchronization has been investigated and studied in physical systems involving real variables over the last 25 years. However, there are also many interesting systems involving complex variables which have not been actively explored.

In this paper, we study the impulsive control and synchronization of complex Lorenz chaotic system described by the following differential equation:

$$
\begin{gathered}
\dot{x}=a(y-x), \quad \dot{y}=c x-y-x z, \\
\dot{z}=-b z+\frac{1}{2}(\bar{x} y+x \bar{y}),
\end{gathered}
$$

where $a, c, b$ are positive (real or complex) parameters, $x$ and $y$ are complex variables, $z$ is a real variable, an over bar denotes complex conjugate variable, and dots represent derivatives with respect to time. This system arises in many important applications in physics, for example, in laser physics and rotating fluids dynamics. These variables have relations with the electric field amplitude, the atomic polarization amplitude, and the population inversion; for more details, see $[2,14,15]$ and references therein.

Numerically we show that this system is chaotic and exhibits chaotic attractors. Analytical and numerical calculations are presented to achieve synchronization. Impulsive control technique is used to synchronize chaotic attractors of (2).

\section{Impulsive Control of a Nonlinear System}

The mathematical description of these impulsive systems of differential equations is usually defined as an ordinary differential equation coupled with a system of difference equations as expressed in the following system:

$$
\begin{gathered}
\dot{x}=f(t, x), \quad t \neq t_{k}, \\
\left.\Delta x\right|_{t_{k}}=x\left(t_{k}^{+}\right)-x\left(t_{k}^{-}\right), \quad t=t_{k}, \\
x\left(t_{0}^{+}\right)=x_{0} \quad(k=1,2,3, \ldots),
\end{gathered}
$$

where $t \in J=\left[t_{0},+\infty\right), t_{0} \geq 0, x \in R^{n}$ is the state variable, and $f: J \times R^{n} \rightarrow R^{n}$ is a continuous-valued function. The impulsive control law of system (3) is given by the sequence $\left\{t_{k}, u_{k}\left(x\left(t_{k}\right)\right)\right\}$, which has the effect of suddenly changing the state of the system at the instants $t_{k}$, where $t_{1}<t_{2}<\cdots<t_{k}<$ $\cdots, \lim _{k \rightarrow \infty} t_{k}=\infty$ and $t_{0}<t_{1}$. The difference equations are given by

$$
\left.\Delta x\right|_{t_{k}}=x\left(t_{k}^{+}\right)-x\left(t_{k}^{-}\right)=u_{k}\left(x\left(t_{k}\right)\right),
$$

where $x\left(t_{k}^{+}\right)=\lim _{t \rightarrow t_{k}^{+}} x(t)$ and $x\left(t_{k}^{-}\right)=\lim _{t \rightarrow t_{k}^{-}} x(t)$. For simplicity, we assume that $x\left(t_{k}^{-}\right)=x\left(t_{k}\right)$ and $u_{k}\left(x\left(t_{k}\right)\right)$ can be chosen as $B_{k} x\left(t_{k}\right)$ where $B_{k}$ is $n \times n$ matrices. The objective is to find some (sufficient) conditions on the constant control gains, $B_{k}$, and the impulsive intervals $\tau_{k}=t_{k}-t_{k-1}<\infty(k=$ $1,2,3, \ldots)$, such that the impulsively controlled system (3) is stable.

2.1. Impulsive Control of the Complex Lorenz System. The complex Lorenz system is described by

$$
\begin{gathered}
\dot{x}=a(y-x), \quad \dot{y}=c x-y-x z, \\
\dot{z}=-b z+\frac{1}{2}(\bar{x} y+x \bar{y}),
\end{gathered}
$$

where $x=x_{1}+i x_{2}, \quad y=x_{3}+i x_{4}$ are complex state variables, $z=x_{5}$ is real state variable, and $a>0, b>0$, and $c>0$ are real parameters (or complex).

The real version of (5) reads

$$
\begin{gathered}
\dot{x}_{1}=a\left(x_{3}-x_{1}\right), \quad \dot{x}_{2}=a\left(x_{4}-x_{2}\right), \\
\dot{x}_{3}=c x_{1}-x_{1} x_{5}-x_{3}, \\
\dot{x}_{4}=c x_{2}-x_{2} x_{5}-x_{4}, \quad \dot{x}_{5}=-b x_{5}+\left(x_{1} x_{3}+x_{2} x_{4}\right) .
\end{gathered}
$$

We can rewrite the above systems into the matrix form:

$$
\left[\begin{array}{c}
\dot{x}_{1} \\
\dot{x}_{2} \\
\dot{x}_{3} \\
\dot{x}_{4} \\
\dot{x}_{5}
\end{array}\right]=\left[\begin{array}{ccccc}
-a & 0 & a & 0 & 0 \\
0 & -a & 0 & a & 0 \\
c & 0 & -1 & 0 & 0 \\
0 & c & 0 & -1 & 0 \\
0 & 0 & 0 & 0 & -b
\end{array}\right]\left[\begin{array}{c}
x_{1} \\
x_{2} \\
x_{3} \\
x_{4} \\
x_{5}
\end{array}\right]+\left[\begin{array}{c}
0 \\
0 \\
-x_{1} x_{5} \\
-x_{2} x_{5} \\
x_{1} x_{3}+x_{2} x_{4}
\end{array}\right] .
$$


The impulsive control of the complex Lorenz system is then given by

$$
\begin{gathered}
\dot{x}=A x+\Phi(x), \quad t \neq t_{k}, \\
\left.\Delta x\right|_{t_{k}}=B_{k} x, \quad t=t_{k}, \\
x\left(t_{0}^{+}\right)=x_{0} \quad(k=1,2,3, \ldots),
\end{gathered}
$$

where $A$ is the linear part matrix of the corresponding system, $\Phi(x)=\left(0,0,-x_{1} x_{5},-x_{2} x_{5}, x_{1} x_{3}+x_{2} x_{4}\right)^{T}$, and $t_{k}$ denotes the instant when impulsive control occurs.

For convenience, define the following notations:

$$
\begin{gathered}
\lambda_{2}(A)=\frac{1}{2} \lambda_{\max }\left(A+A^{T}\right), \\
\beta_{k}=\lambda_{\max }\left[\left(I+B_{k}\right)^{T}\left(I+B_{k}\right)\right],
\end{gathered}
$$

where $I$ is the $n \times n$ identity matrix and $\lambda_{\max }(Q)$ is the maximal eigenvalue of matrix $Q$.

Theorem 1. (I) If $2 \lambda_{2}(A)=\lambda<0$ ( $\lambda$ is a constant) and there exists a constant $0 \leq \alpha<-\lambda$, such that

$$
\ln \beta_{k}-\alpha\left(t_{k}-t_{k-1}\right) \leq 0, \quad k=1,2, \ldots,
$$

then the trivial solution of system (8) is globally exponentially stable.

(II) If $2 \lambda_{2}(A)=\lambda \geq 0$ ( $\lambda$ is a constant) and there exists $a$ constant $\alpha \geq 1$, such that

$$
\ln \left(\alpha \beta_{k}\right)+\lambda\left(t_{k}-t_{k-1}\right) \leq 0, \quad k=1,2, \ldots,
$$

then $\alpha=1$ implies that the trivial solution of system (8) is stable and $\alpha>1$ implies that the trivial solution of system (8) is globally asymptotically stable.

Notes 1 . Theorem 1 gives sufficient conditions for the global exponential stability and global asymptotical stability for controlling the systems to the origin. The results are new and comprehensive for the impulsive control of the Lorenz systems family. Also, the conditions imply that the impulsive intervals may not be equidistant.

Corollary 2. Assume that $\tau_{k}=\tau>0$ and matrices $B_{k}=$ $B(k=1,2, \ldots)$.

(i) If $2 \lambda_{2}(A)=\lambda<0$ ( $\lambda$ is a constant) and there exists $a$ constant $0 \leq \alpha<-\lambda$, such that $\ln \beta-\alpha \tau \leq 0$, then the trivial solution of system (8) is globally exponentially stable.

(ii) If $2 \lambda_{2}(A)=\lambda \geq 0$, ( $\lambda$ is a constant) and there exists a constant $\alpha \geq 1$, such that $\ln (\alpha \beta)+\lambda \tau \leq 0$, then the conclusion of Theorem 1 holds.

2.2. Impulsive Synchronization of the Complex Lorenz System. We study the impulsive synchronization of the complex Lorenz system. For simplifying the problem, we assume that we have two identical complex Lorenz systems and denote the drive system by the subscript $d$, while the response system to be controlled is denoted by the subscript $r$. Our aim is to design an impulsive controller and make the response system follow the drive system and become ultimately the same. The drive and response systems are defined, respectively, as

$$
\begin{gathered}
\dot{x}_{d}=a\left(y_{d}-x_{d}\right), \quad \dot{y}_{d}=c x_{d}-y_{d}-x_{d} z_{d}, \\
\dot{z}_{d}=-b z_{d}+\frac{1}{2}\left(\bar{x}_{d} y_{d}+x_{d} \bar{y}_{d}\right), \\
\dot{x}_{r}=a\left(y_{r}-x_{r}\right), \quad \dot{y}_{r}=c x_{r}-y_{r}-x_{r} z_{r}, \\
\dot{z}_{r}=-b z_{r}+\frac{1}{2}\left(\bar{x}_{r} y_{r}+x_{r} \bar{y}_{r}\right),
\end{gathered}
$$

where $x_{d}=x_{1 d}+i x_{2 d}, \quad y_{d}=x_{3 d}+i x_{4 d}$ are complex state variables, $z_{d}=x_{5 d}$ is real state variable, $x_{r}=x_{1 r}+i x_{2 r}, y_{r}=$ $x_{3 r}+i x_{4 r}, z_{r}=x_{5 r}$, and $\left(\begin{array}{c}- \\ \ldots\end{array}\right)$ denotes the complex conjugate.

The complex systems (12) and (13) can be rewritten, respectively, as five real first-order ODEs of the form

$$
\begin{aligned}
& \dot{x}_{1 d}=a\left(x_{3 d}-x_{1 d}\right), \\
& \dot{x}_{2 d}=a\left(x_{4 d}-x_{2 d}\right), \\
& \dot{x}_{3 d}=c x_{1 d}-x_{3 d}-x_{1 d} x_{5 d}, \\
& \dot{x}_{4 d}=c x_{2 d}-x_{4 d}-x_{2 d} x_{5 d}, \\
& \dot{x}_{5 d}=-b x_{5 d}+\left(x_{1 d} x_{3 d}+x_{2 d} x_{4 d}\right), \\
& \dot{x}_{1 r}=a\left(x_{3 r}-x_{1 r}\right), \\
& \dot{x}_{2 r}=a\left(x_{4 r}-x_{2 r}\right), \\
& \dot{x}_{3 r}=c x_{1 r}-x_{3 r}-x_{1 r} x_{5 r}, \\
& \dot{x}_{4 r}=c x_{2 r}-x_{4 r}-x_{2 r} x_{5 r}, \\
& \dot{x}_{5 r}=-b x_{5 r}+\left(x_{1 r} x_{3 r}+x_{2 r} x_{4 r}\right) .
\end{aligned}
$$

We define the error states between the response system that is controlled and the controlling driving system as

$$
\begin{gathered}
e_{x_{1}}+i e_{x_{2}}=x_{r}-x_{d}=\left(x_{1 r}-x_{1 d}\right)+i\left(x_{2 r}-x_{2 d}\right), \\
e_{x_{3}}+i e_{x_{4}}=y_{r}-y_{d}=\left(x_{3 r}-x_{3 d}\right)+i\left(x_{4 r}-x_{4 d}\right), \\
e_{x_{5}}=z_{r}-z_{d}=x_{5 r}-x_{5 d} .
\end{gathered}
$$

In an impulsive configuration, the driving system and the response system are modeled by the following impulsive equations:

$$
\begin{gathered}
\dot{x}_{d}=A x_{d}+\Phi\left(x_{d}\right), \quad t \neq t_{k}, \\
\left.\Delta x_{d}\right|_{t_{k}}=B_{k} x_{d}, \quad t=t_{k}, \\
x_{d}\left(t_{0}^{+}\right)=x_{d}\left(t_{0}\right) \quad(k=1,2,3, \ldots), \\
\dot{x}_{r}=A x_{r}+\Phi\left(x_{r}\right), \quad t \neq t_{k}, \\
\left.\Delta x_{r}\right|_{t_{k}}=B_{k} x_{r}, \quad t=t_{k}, \\
x_{r}\left(t_{0}^{+}\right)=x_{r}\left(t_{0}\right) \quad(k=1,2,3, \ldots) .
\end{gathered}
$$




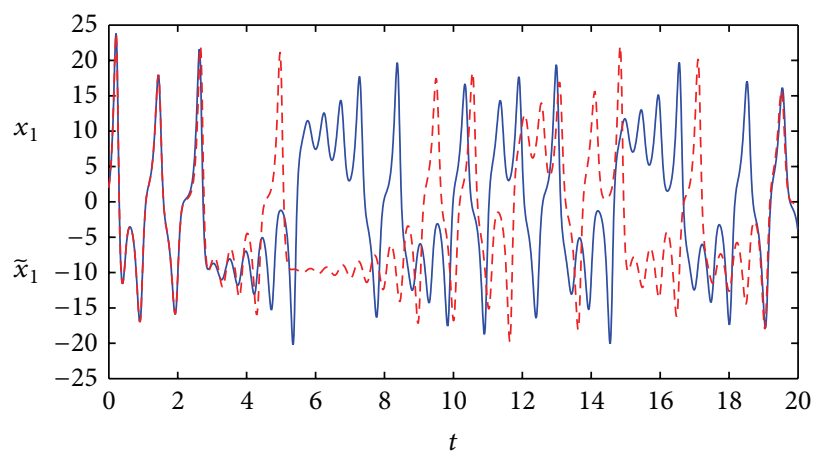

(a)

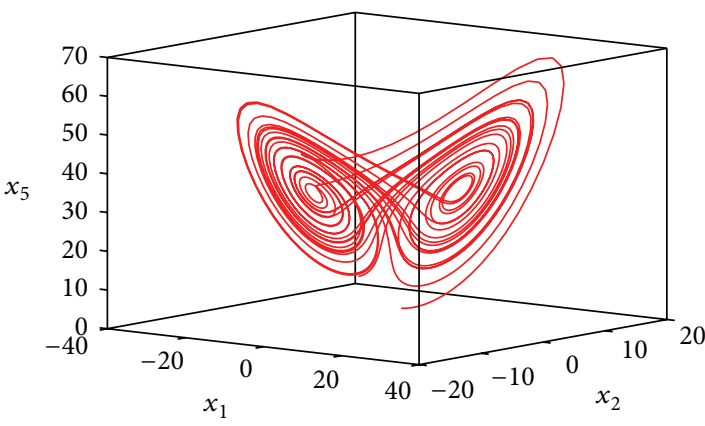

(b)

Figure 1: Left figure: Two numerical solutions of (5) for $a=14 ; c=35 ; b=3.7$ with $t_{0}=0 ; x_{1}(0)=2 ; x_{2}(0)=1 ; x_{3}(0)=5 ; x_{4}(0)=3$; $x_{5}(0)=4$ (the solid curve) and $\tilde{x}_{1}(0)=2.001 ; \tilde{x}_{2}(0)=1 ; \tilde{x}_{3}(0)=5 ; \tilde{x}_{4}(0)=3 ; \tilde{x}_{5}(0)=4.01$ (the dotted curve) $(t=$ time/10). Right figure: Chaotic attractor of $(5)$ at $a=14 ; c=35 ; b=3.7$ in $\left(x_{1} ; x_{2} ; x_{5}\right)$ space.

If we defined

$$
\begin{aligned}
\Psi\left(x_{r}, x_{d}\right) & =\Phi\left(x_{r}\right)-\Phi\left(x_{d}\right) \\
& =\left[\begin{array}{c}
0 \\
0 \\
-x_{r_{1}} x_{r_{5}}+x_{d_{1}} x_{d_{5}} \\
-x_{r_{2}} x_{r_{5}}+x_{d_{2}} x_{d_{5}} \\
x_{r_{1}} x_{r_{3}}+x_{r_{2}} x_{r_{4}}-x_{d_{1}} x_{d_{3}}-x_{d_{2}} x_{d_{4}}
\end{array}\right],
\end{aligned}
$$

then the error system of the impulsive synchronization is given by

$$
\begin{gathered}
\dot{e}=A e+\Psi\left(x_{r}, x_{d}\right), \quad t \neq t_{k}, \\
\left.\Delta e\right|_{t_{k}}=B_{k} e, \quad t=t_{k}, \\
e\left(t_{0}^{+}\right)=x_{r}\left(t_{0}\right)-x_{d}\left(t_{0}\right) \quad(k=1,2,3, \ldots) .
\end{gathered}
$$

Note that there exists a positive constant $M$ for the chaotic system (6) such that $|x(t)| \leq M$ for all $t$. For convenience, define the following notations:

$$
\begin{gathered}
\lambda_{2}(A)=\frac{1}{2} \lambda_{\max }\left(A+A^{T}\right), \\
\beta_{k}=\lambda_{\max }\left[\left(I+B_{k}\right)^{T}\left(I+B_{k}\right)\right] .
\end{gathered}
$$

Notes 2. From the analysis above, it follows that a necessary and sufficient condition for synchronizing chaos is that the origin of (20) is asymptotically stable. It is obvious that the origin is one of the equilibriums of (20). Also, the origin is the unique equilibrium of (20) because $\left.\Delta e\right|_{t_{k}}=B_{k} e, \quad t=t_{k}$.

Theorem 3. (I) If $2 \lambda_{2}(A)+2 M=\lambda<0$ ( $\lambda$ is a constant) and there exists a constant $0 \leq \alpha<-\lambda$, such that $\ln \beta_{k}-\alpha\left(t_{k}-\right.$ $\left.t_{k-1}\right) \leq 0, k=1,2, \ldots$, then the trivial solution of system (20) is globally exponentially stable; that is, system (18) is globally exponentially synchronous with system (17).

(II) If $2 \lambda_{2}(A)+2 M=\lambda \geq 0$ ( $\lambda$ is a constant) and there exists a constant $\alpha \geq 1$, such that $\ln \left(\alpha \beta_{k}\right)+\lambda\left(t_{k}-t_{k-1}\right) \leq 0, k=$
$1,2, \ldots$, then system (18) is globally exponentially synchronous with system (17).

Corollary 4. Assume that $t_{k}=\tau>0$ and matrices $B_{k}=B$ $(k=1,2, \ldots)$.

(i) If $2 \lambda_{2}(A)+2 M=\lambda<0$ and there exists a constant $0 \leq \alpha<-\lambda$, such that $\ln \beta-\alpha \tau \leq 0$, then the system (18) is globally exponentially synchronous with system (17).

(ii) If $2 \lambda_{2}(A)+2 M=\lambda \geq 0$ ( $\lambda$ is a constant) and there exists a constant $\alpha \geq 1$, such that $\ln (\alpha \beta)+\lambda \tau \leq 0$, $k=1,2, \ldots$, then system (18) is globally exponentially synchronous with system (17).

\section{Simulation Results}

In this simulation, the chaotic complex Lorenz system will be taken, for example, to confirm the proposed method.

The equilibria of (6) can be calculated by solving the equations $\dot{x}_{1}=0, \dot{x}_{2}=0, \dot{x}_{3}=0, \dot{x}_{4}=0$, and $\dot{x}_{5}=0$ to get three equilibria $E_{0}=(0,0,0,0,0), E_{1,2}=$ $\left( \pm x_{1}^{*}, \pm x_{2}^{*}, \pm x_{3}^{*}, \pm x_{4}^{*}, x_{5}^{*}\right)$, where

$$
x_{1}^{*}=x_{2}^{*}=x_{3}^{*}=x_{4}^{*}=\sqrt{\frac{b(c-1)}{2}}, \quad x_{5}^{*}=(c-1) .
$$

For $c>1$, the nontrivial equilibria exist.

The eigenvalues of the corresponding linearized system at $E_{0}$ are

$$
\begin{gathered}
\lambda_{1}=-b, \\
\lambda_{2}=\lambda_{3}=-\left(\frac{a-1}{2}\right)+\sqrt{\left(\frac{a-1}{2}\right)^{2}+a(c-1)}, \\
\lambda_{4}=\lambda_{5}=-\left(\frac{a-1}{2}\right)-\sqrt{\left(\frac{a-1}{2}\right)^{2}+a(c-1) .}
\end{gathered}
$$

The equilibrium $E_{0}$ is stable if $c<1$ and it becomes unstable if $c>1$. 


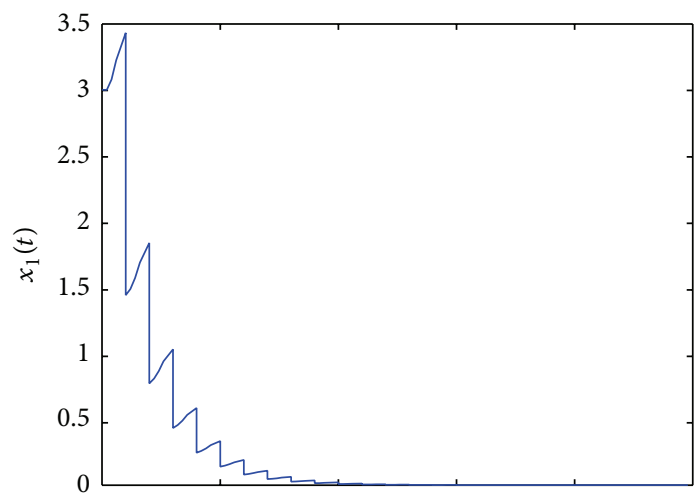

(a)

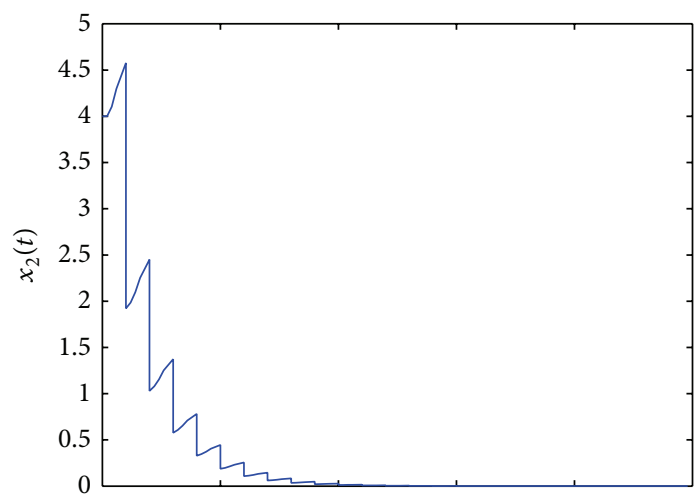

(c)

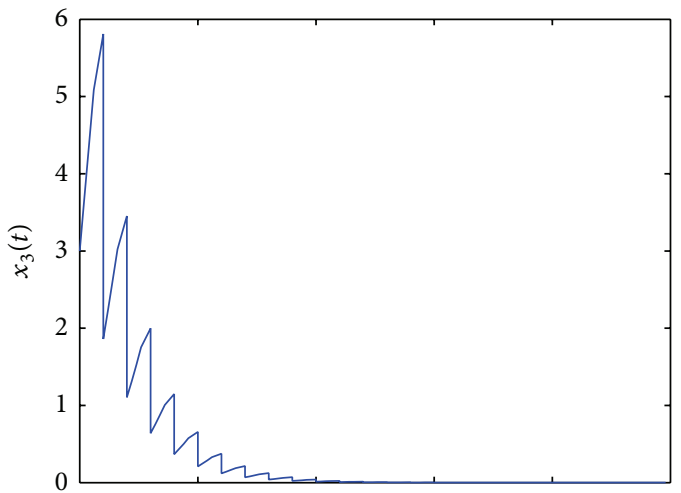

(e)

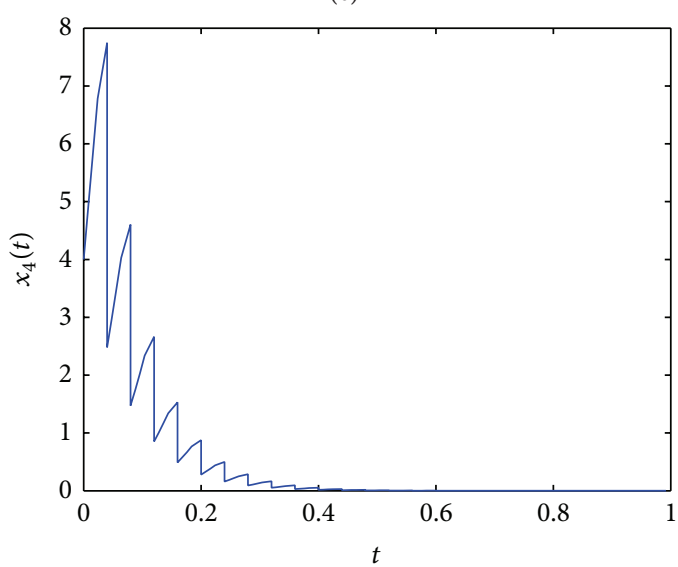

(g)

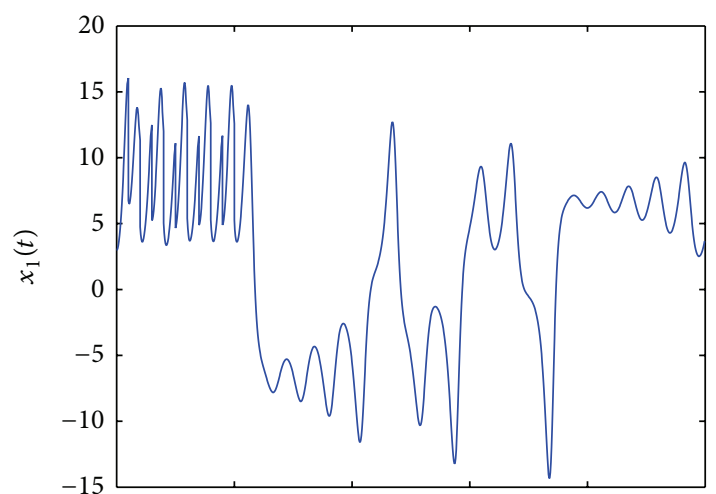

(b)

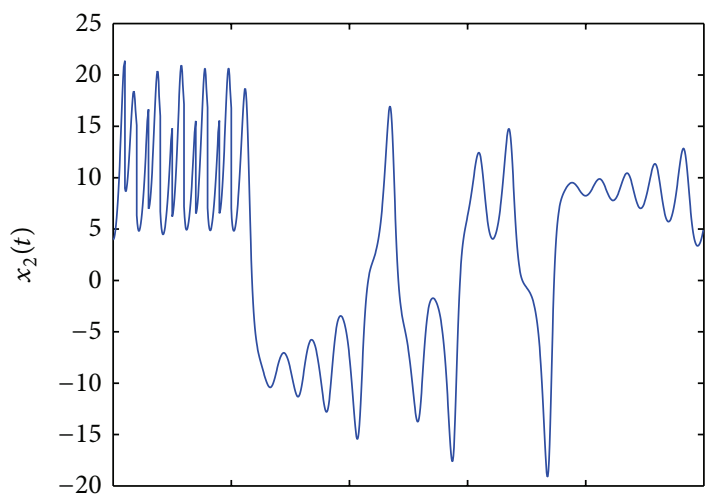

(d)

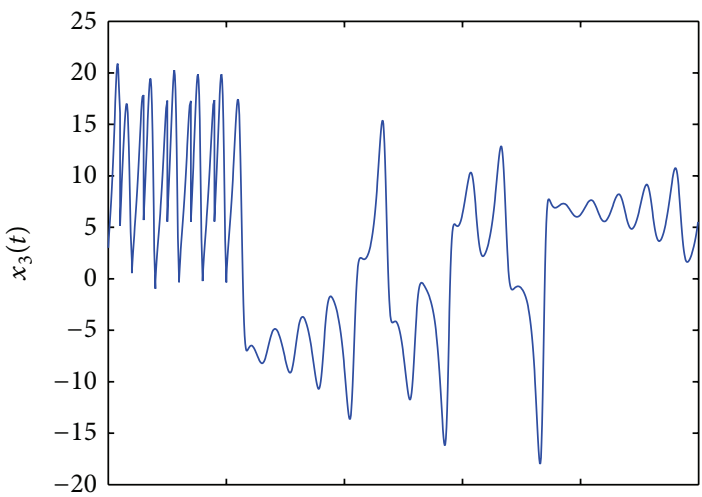

(f)

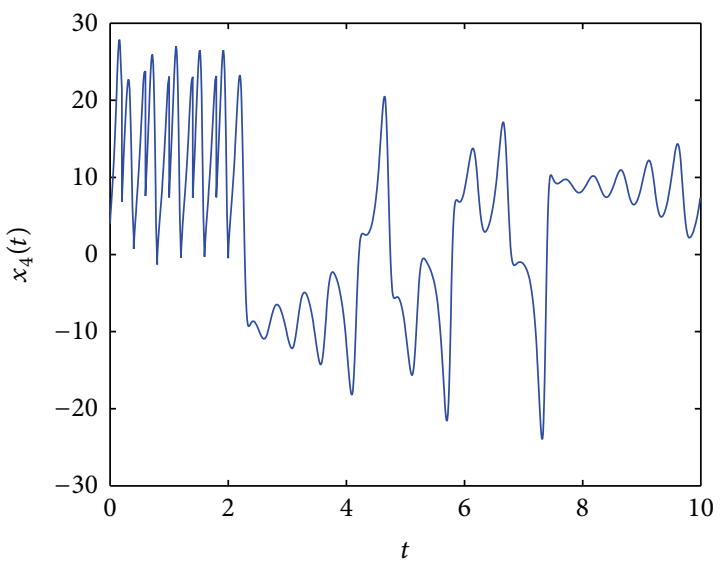

(h)

Figure 2: Continued. 


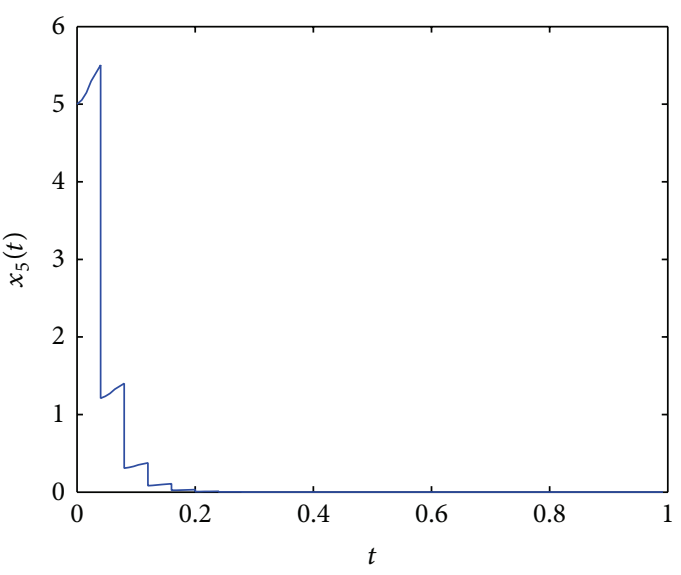

(i)

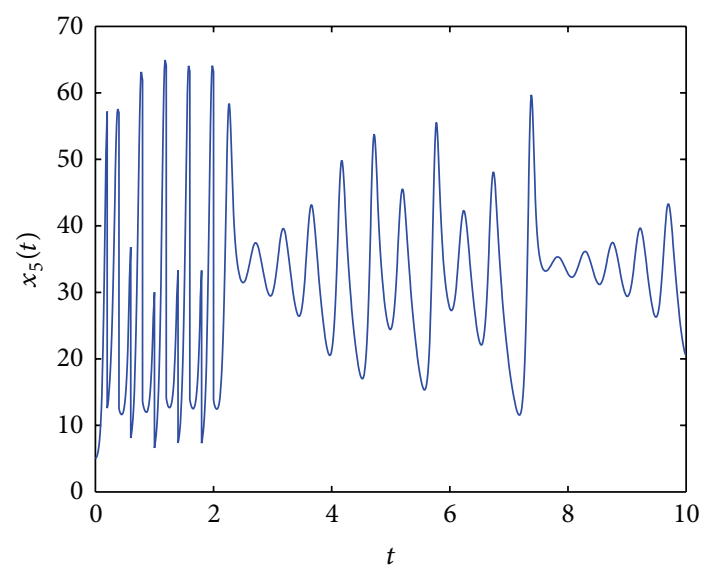

(j)

FIGURE 2: Left figures: Impulsively control complex Lorenz system with $\tau=0.01$, Right figures: Impulsively control complex Lorenz system cannot be stabilized with $\tau=0.2$.

Solving the system (6) (or (5)) numerically, we show that system (6) is a chaotic system since the separation of two nearby trajectories increases exponentially with time as in Figure 1. In this figure we show two numerical solutions of (6) with two close initial conditions $x_{1}(0)=2, x_{2}(0)=1$, $x_{3}(0)=5, x_{4}(0)=3, x_{5}(0)=4$ and $\tilde{x}_{1}(0)=2.001$, $\tilde{x}_{2}(0)=1, \tilde{x}_{3}(0)=2, \tilde{x}_{4}(0)=3, \tilde{x}_{5}(0)=4.01$ (we plot only $\left(t, x_{1}\right)$ diagram) and with $a=14, c=35$, and $b=3.7$.

It is clear from Figure 1 that our system displays sensitive dependence on initial conditions.

The system (6) exhibits chaotic attractor for $a=14$, $c=35$, and $b=3.7$ with the initial conditions $t_{0}=0$; $x_{1}(0)=2, x_{2}(0)=1, x_{3}(0)=5, x_{4}(0)=3$, and $x_{5}(0)=4$; see Figure 1 in $\left(x_{1}, x_{2}, x_{5}\right)$ space, respectively. The projection of the chaotic attractor of $(6)$ in $\left(x_{1}, x_{2}, x_{5}\right)$ space is similar to Lorenz attractor of (1) as in [2].

We control system (6) to its equilibrium point $(0,0,0,0,0)^{T}$; thus

$$
\begin{aligned}
A+A^{T} & =\left[\begin{array}{ccccc}
-2 a & 0 & a+c & 0 & 0 \\
0 & -2 a & 0 & a+c & 0 \\
a+c & 0 & -2 & 0 & 0 \\
0 & a+c & 0 & -2 & 0 \\
0 & 0 & 0 & 0 & -2 \beta
\end{array}\right] \\
& =\left[\begin{array}{ccccc}
-28 & 0 & 49 & 0 & 0 \\
0 & -28 & 0 & 49 & 0 \\
49 & 0 & -2 & 0 & 0 \\
0 & 49 & 0 & -2 & 0 \\
0 & 0 & 0 & 0 & -7.4
\end{array}\right] .
\end{aligned}
$$

Its eigenvalues are $-65.69516742,-65.69516742$, $7.400000000,35.69516742$, and 35.69516742. Then $2 \lambda_{2}(A)=$ $\lambda=35.69516742 \geq 0$. If we choose $B=\operatorname{diag}\left(b_{1}, b_{2}, b_{3}, b_{4}\right.$, $\left.b_{5}\right)=\operatorname{diag}(-0.67,-0.67,-0.77,-0.77,-0.87)$, then

$$
\begin{aligned}
\beta & =\max \left\{\left(1+b_{1}\right)^{2},\left(1+b_{2}\right)^{2},\left(1+b_{3}\right)^{2},\left(1+b_{4}\right)^{2},\left(1+b_{5}\right)^{2}\right\} \\
& =0.1764 .
\end{aligned}
$$

If $\tau<-(\ln \alpha \beta / \lambda)$, from Theorem 1 , we have that the equilibrium point of the impulsively controlled system is asymptotically stable. Take $\alpha=1.01$; thus if $\tau<0.04832729273$, system (6) will be stabilized at the origin. Let $\tau=0.01$, respectively; the simulation results are shown in Figure 2, where the initial conditions are $(3.0,4.0,3.0,4.0,5.0)^{T}$. From Figure 2 we have that the state variables quickly tend to the origin under impulsive control. If the impulse intervals are too large, as proved previously, the impulsively controlled system cannot be stabilized, as shown in Figure 2 with $\tau=$ 0.2 .

In Figure 3, we show the error synchronization of two complex Lorenz systems with the following initial conditions for the drive and response systems are chosen as $(3.0,4.0,3.0,4.0,5.0)^{T}$ and $(6.0,7.0,6.0,7.0,8.0)^{T}$. We can get from the simulation that the approximate bounds $M$ of system (6) are 85 . Thus $\lambda=2 \lambda_{2}\left(A_{0}\right)+2 M=35.69516742+$ $2 M=115.69516742$. Choose $B=\operatorname{diag}\left(b_{1}, b_{2}, b_{3}, b_{4}, b_{5}\right)=$ $(-0.67,-0.67,-0.77,-0.77,-0.78)$. Take $\alpha=1.01$; thus if $\tau<-(\ln \alpha \beta / \lambda)=0.019$, system (17) is globally asymptotically synchronous with system (6). Figure 3 shows the results when $\tau=0.01$. It is obvious that, with this impulsive control, two chaotic systems synchronize very fast.

\section{Conclusions}

Our interest in the present paper is to study the impulsive control and synchronization of chaotic attractors of the complex Lorenz system (5). This study can be considered as a continuation of our studies in the literature for complex 


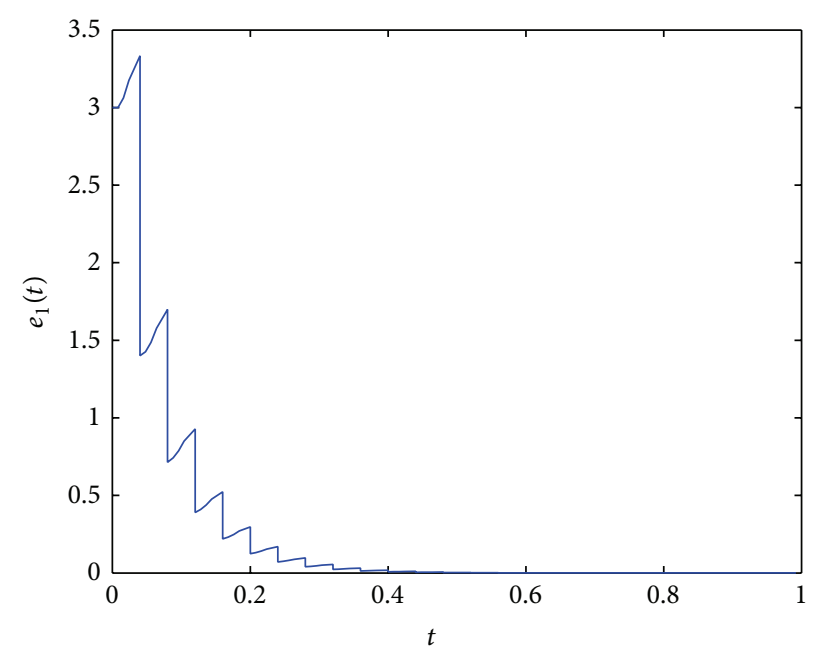

(a)

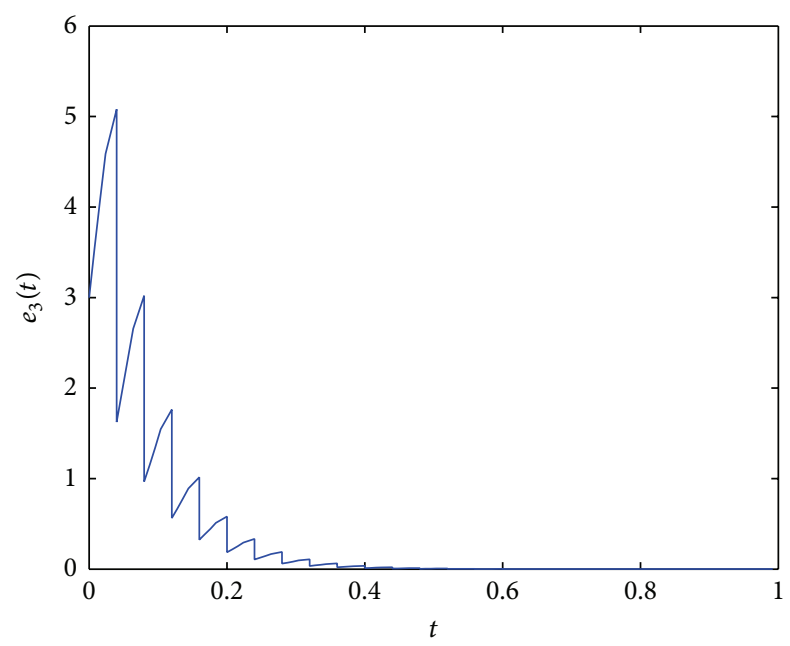

(c)

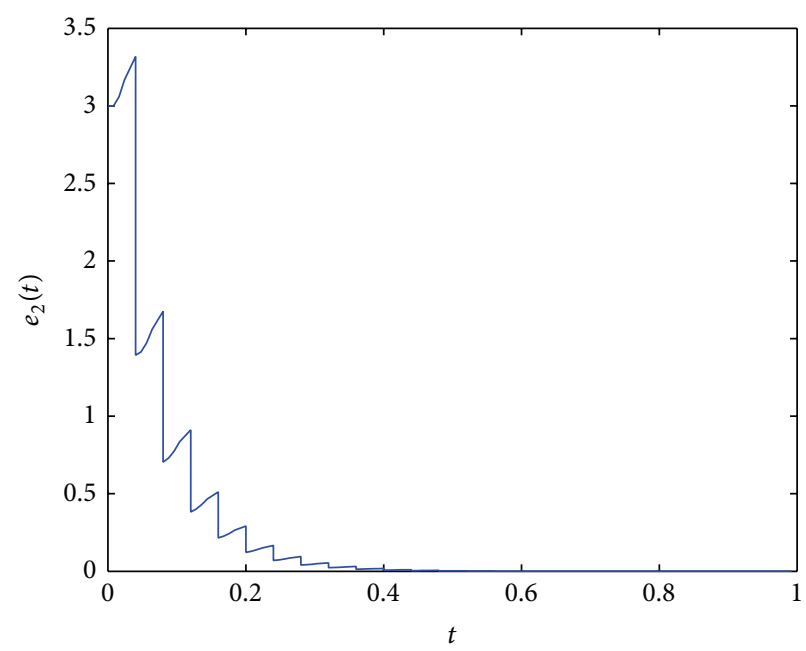

(b)

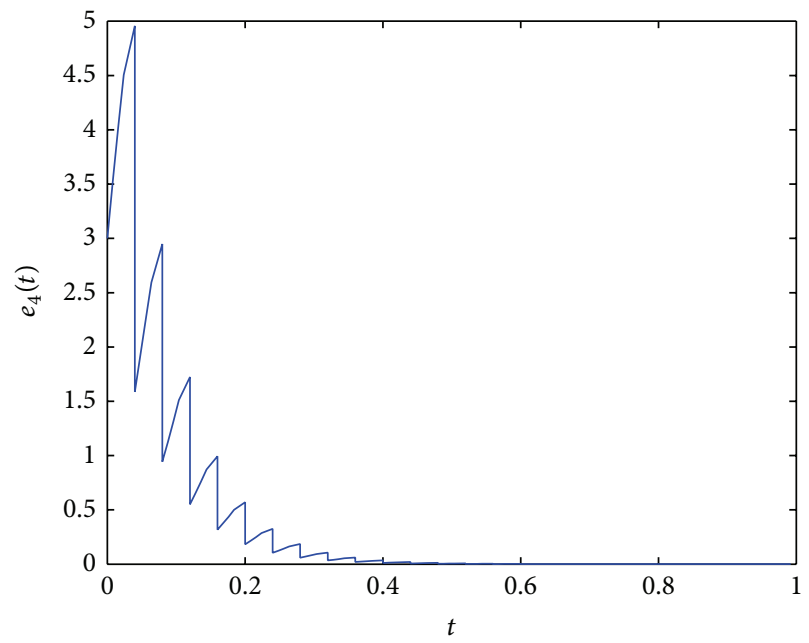

(d)

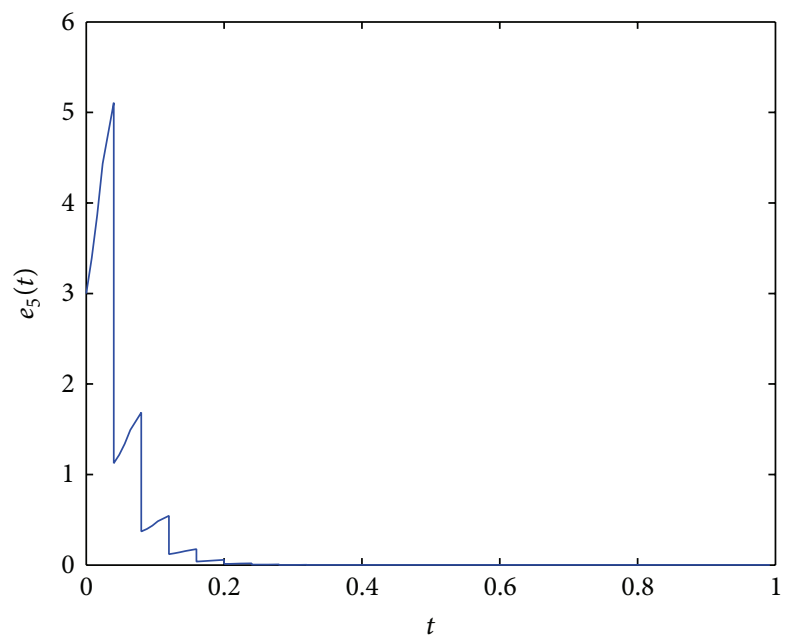

(e)

FIGURE 3: Synchronization errors solutions of system (20) with $\tau=0.01$. 
Lorenz systems. These complex systems arise in several important fields of physics, for example, laser physics, rotating fluids dynamics, and disk dynamos. We define chaos as sensitive dependence on initial conditions and measure it by computing the rate of separation of two initially nearby trajectories in phase space. System (5) is a chaotic system and exhibits chaotic attractors. Sensitive dependence on initial conditions and on the system's parameters is prominent characteristic of chaotic behavior of (5). The projection of the chaotic attractor of $(6)$ in $\left(x_{1}, x_{2}, x_{5}\right)$ space is similar to Lorenz attractor with real variables (1). Some new and more comprehensive criteria for global exponential stability and asymptotical stability of impulsively controlled complex Lorenz systems are established with varying impulsive intervals. The effectiveness of the proposed technique is verified through numerical simulations, and it can be seen that the synchronization errors $e_{x_{i}}$ converge to zero, as shown in Figure 3.

\section{Conflict of Interests}

The authors declare that there is no conflict of interests regarding the publication of this paper.

\section{Acknowledgment}

The authors are very grateful to all members of Applied Mathematics Group (Department of Mathematics, Faculty of Science, King Khalid University, Saudi Arabia) for useful discussions on the topics investigated in this paper.

\section{References}

[1] G. M. Mahmoud, S. A. Aly, and M. A. AL-Kashif, "Dynamical properties and chaos synchronization of a new chaotic complex nonlinear system," Nonlinear Dynamics, vol. 51, no. 1-2, pp. 171181, 2008.

[2] G. M. Mahmoud, M. A. Al-Kashif, and S. A. Aly, "Basic properties and chaotic synchronization of complex Lorenz system," International Journal of Modern Physics C: Computational Physics, Physical Computation, vol. 18, no. 2, pp. 253-265, 2007.

[3] G. M. Mahmoud and T. Bountis, "The dynamics of systems of complex nonlinear oscillators: a review," International Journal of Bifurcation and Chaos in Applied Sciences and Engineering, vol. 14, no. 11, pp. 3821-3846, 2004.

[4] G. M. Mahmoud, "Approximate solutions of a class of complex nonlinear dynamical systems," Physica A: Statistical Mechanics and its Applications, vol. 253, no. 1-4, pp. 211-222, 1998.

[5] M. Schmutz and M. Rueff, "Bifurcation schemes of the Lorenz model," Physica D: Nonlinear Phenomena, vol. 11, no. 1-2, pp. 167-178, 1984.

[6] A. C. Fowler, J. D. Gibbon, and M. J. McGuinness, "The real and complex Lorenz equations and their relevance to physical systems," Physica D: Nonlinear Phenomena, vol. 7, no. 1-3, pp. 126-134, 1983.

[7] C.-Z. Ning and H. Haken, "Detuned lasers and the complex Lorenz equations: subcritical and supercritical Hopf bifurcations," Physical Review A, vol. 41, no. 7, pp. 3826-3837, 1990.

[8] A. G. Vladimirov, V. Yu. Toronov, and V. L. Derbov, "The complex Lorenz model: geometric structure, homoclinic bifurcation and one-dimensional map," International Journal of Bifurcation and Chaos in Applied Sciences and Engineering, vol. 8, no. 4, pp. 723-729, 1998.

[9] C. A. Jones, N. O. Weiss, and F. Cattaneo, "Nonlinear dynamos: a complex generalization of the Lorenz equations," Physica D: Nonlinear Phenomena, vol. 14, no. 2, pp. 161-176, 1985.

[10] G. P. Flessas, "New exact solutions of the complex Lorenz equations," Journal of Physics A: Mathematical and General, vol. 22, no. 5, pp. L137-L141, 1989.

[11] P. H. Roberts and G. A. Glazmaier, "Geodynamo theory and simulations," Reviews of Modern Physics, vol. 72, pp. 1083-1123, 2000.

[12] S. Panchev and N. K. Vitanov, "On asymptotic properties of some complex Lorenz-like systems," Journal of the Calcutta Mathematical Society, vol. 1, no. 3-4, pp. 181-190, 2005.

[13] V. Yu. Toronov and V. L. Derbov, "Boundedness of attractors in the complex Lorenz model," Physical Review E: Statistical, Nonlinear, and Soft Matter Physics, vol. 55, no. 3, pp. 3689-3692, 1997.

[14] D. J. Tritton, Physical Fluid Dynamics, Oxford Science Publications, The Clarendon Press, Oxford University Press, New York, NY, USA, 2nd edition, 1988.

[15] A. Rauh, L. Hannibal, and N. B. Abraham, "Global stability properties of the complex Lorenz model," Physica D: Nonlinear Phenomena, vol. 99, no. 1, pp. 45-58, 1996.

[16] J. A. Yorke and E. D. Yorke, "Metastable chaos: the transition to sustained chaotic behavior in the Lorenz model," Journal of Statistical Physics, vol. 21, no. 3, pp. 263-277, 1979.

[17] T. Shimizu and N. Morioka, "Transient behavior in periodic regions of the Lorenz model," Physics Letters A, vol. 69, no. 3, pp. 148-150, 1978.

[18] L. M. Pecora and T. L. Carroll, "Synchronization in chaotic systems," Physical Review Letters, vol. 64, no. 8, pp. 821-824, 1990.

[19] G. M. Mahmoud, A. Rauh, and A. A. Mohamed, "On modulated complex non-linear dynamical systems," Il Nuovo Cimento della Società Italiana di Fisica B: Serie 12, vol. 114, no. 1, pp. 31-47, 1999.

[20] G. M. Mahmoud, A. Rauh, and A. A. M. Farghaly, "Applying chaos control to a modulated complex nonlinear system," Nuovo Cimento della Societa Italiana di Fisica B, vol. 116, no. 10, pp. 113126, 2001.

[21] E. Ott, C. Grebogi, and J. A. Yorke, "Controlling chaos," Physical Review Letters, vol. 64, no. 11, pp. 1196-1199, 1990.

[22] J. Lu, X. Wu, and J. Lü, "Synchronization of a unified chaotic system and the application in secure communication," Physics Letters A, vol. 305, no. 6, pp. 365-370, 2002.

[23] G. M. Mahmoud and S. A. Aly, "Periodic attractors of complex damped non-linear systems," International Journal of NonLinear Mechanics, vol. 35, no. 2, pp. 309-323, 2000.

[24] G. M. Mahmoud, S. A. Aly, and A. A. Farghaly, "On chaos synchronization of a complex two coupled dynamos system," Chaos, Solitons and Fractals, vol. 33, no. 1, pp. 178-187, 2007.

[25] T.-L. Liao, "Adaptive synchronization of two Lorenz systems," Chaos, Solitons and Fractals, vol. 9, no. 9, pp. 1555-1561, 1998.

[26] T.-L. Liao and S.-H. Lin, "Adaptive control and synchronization of Lorenz systems," Journal of the Franklin Institute: Engineering and Applied Mathematics, vol. 336, no. 6, pp. 925-937, 1999.

[27] J. Hu, Z. Wang, B. Shen, and H. Gao, "Quantised recursive filtering for a class of nonlinear systems with multiplicative noises and missing measurements," International Journal of Control, vol. 86, no. 4, pp. 650-663, 2013. 
[28] J. Hu, D. Chen, and J. Du, "State estimation for a class of discrete nonlinear systems with randomly occurring uncertainties and distributed sensor delays," International Journal of General Systems, vol. 43, no. 3-4, pp. 387-401, 2014.

[29] J. Hu, Z. Wang, and H. Gao, "Recursive filtering with random parameter matrices, multiple fading measurements and correlated noises," Automatica, vol. 49, no. 11, pp. 3440-3448, 2013.

[30] H. Richter, "Controlling the Lorenz system: combining global and local schemes," Chaos, Solitons and Fractals, vol. 12, no. 13, pp. 2375-2380, 2001.

[31] I. Stewart, “The Lorenz attractor exists" Nature, vol. 406, pp. 948-949, 2000.

[32] V. Lakshmikantham, D. D. Bainnov, and P. S. Simeonov, Theory of Impulsive Differential Equations, vol. 6 of Series in Modern Applied Mathematics, World Scientific Publishing, Singapore, 1989.

[33] T. Yang, L.-B. Yang, and C.-M. Yang, "Impulsive control of Lorenz system," Physica D: Nonlinear Phenomena, vol. 110, no. 1-2, pp. 18-24, 1997.

[34] S. Chen, Q. Yang, and C. Wang, "Impulsive control and synchronization of unified chaotic system," Chaos, Solitons and Fractals, vol. 20, no. 4, pp. 751-758, 2004. 


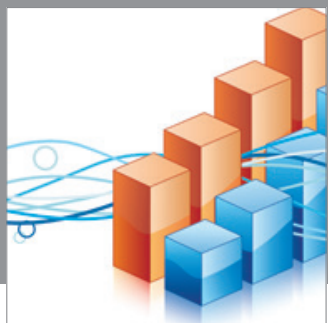

Advances in

Operations Research

mansans

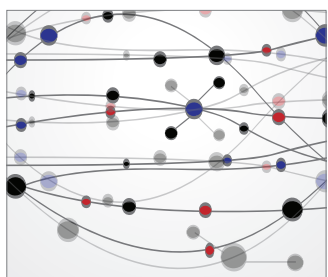

The Scientific World Journal
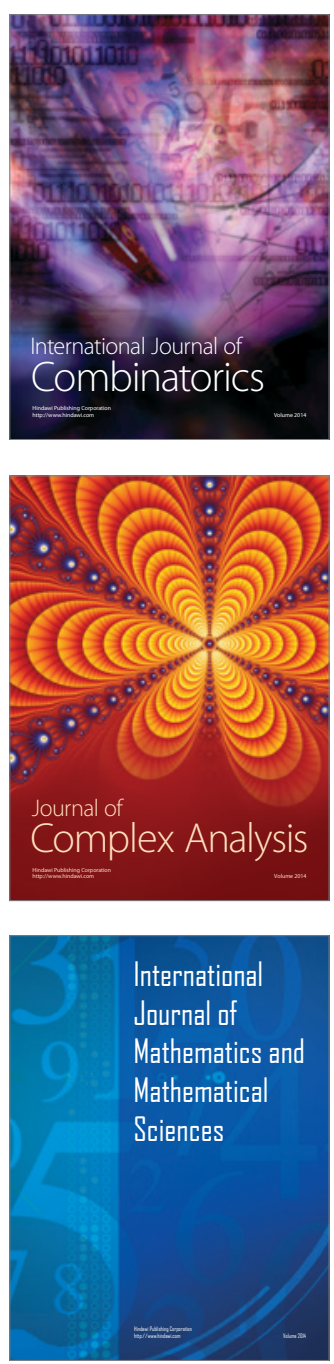
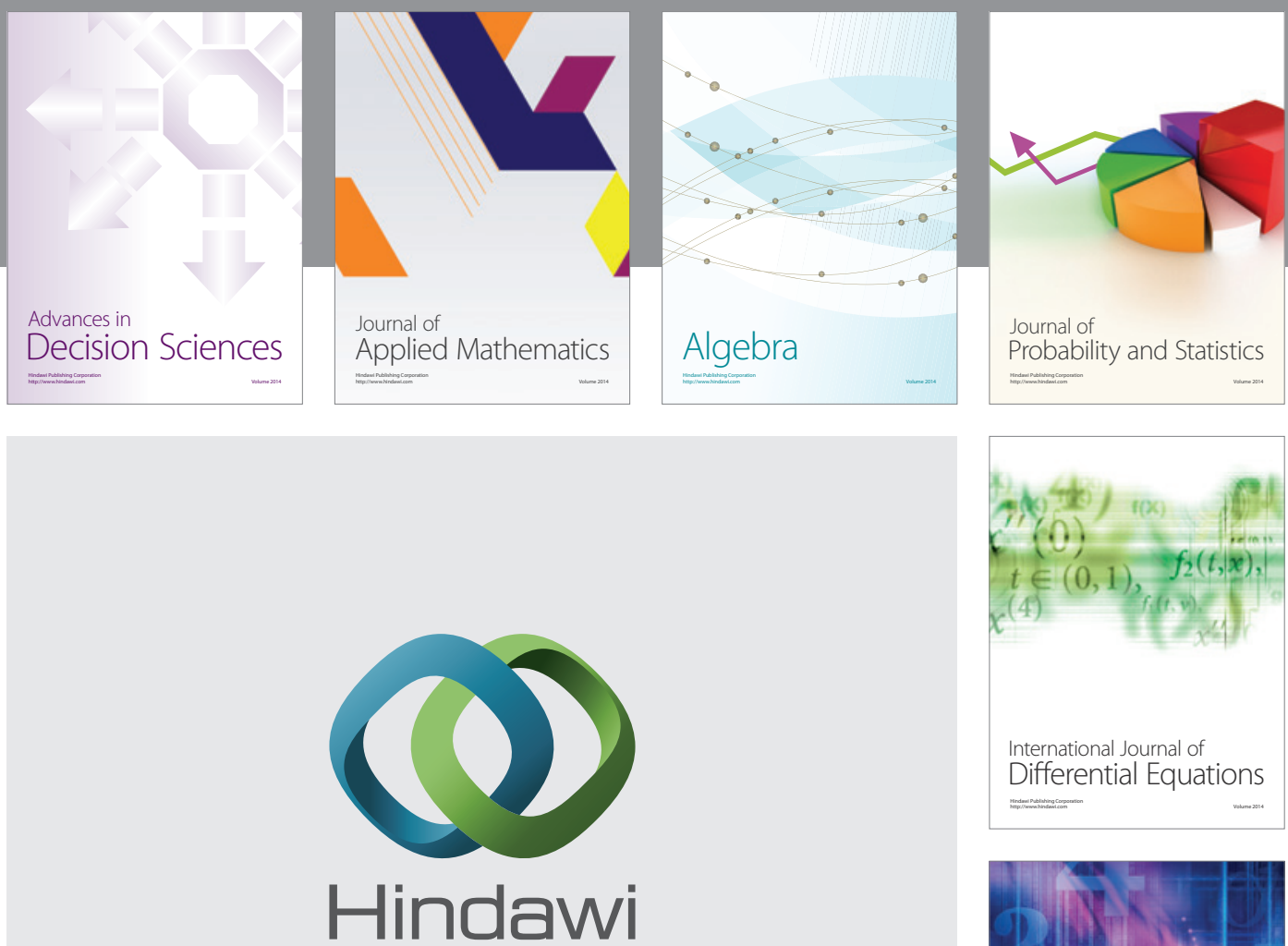

Submit your manuscripts at http://www.hindawi.com
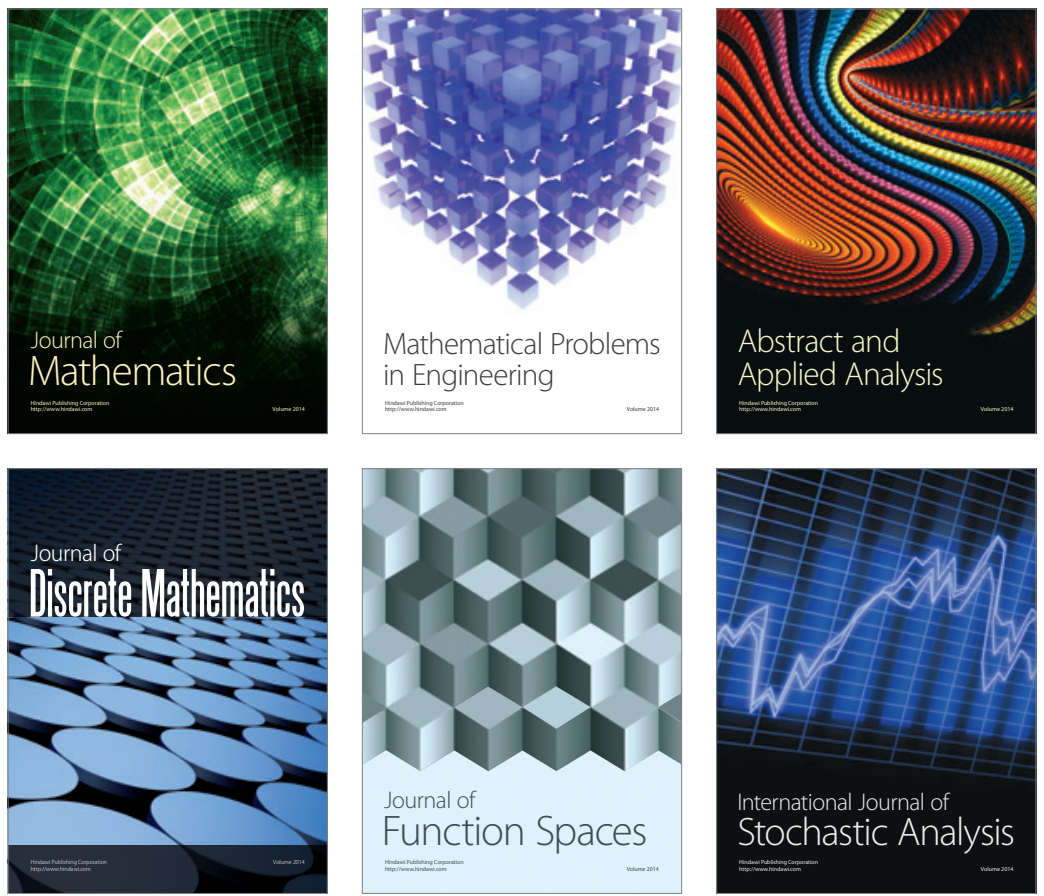

Journal of

Function Spaces

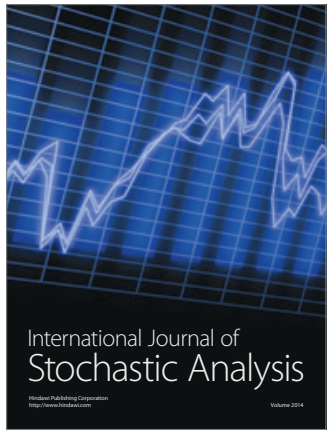

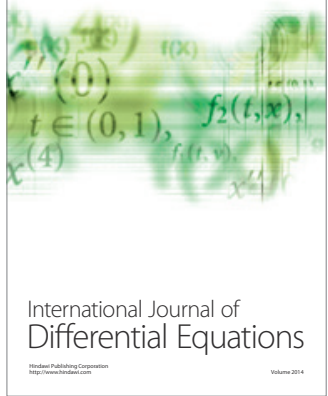
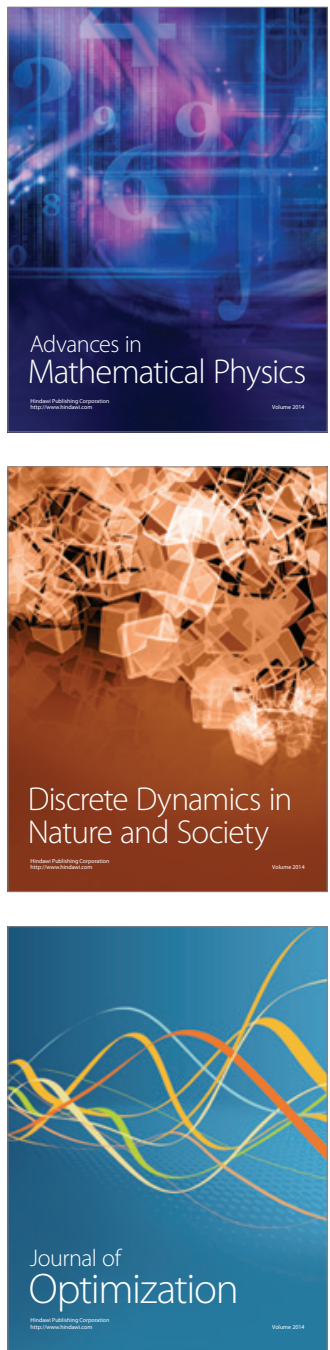